\title{
TECNO TRANS UNA PYME HACIA LA MANUFACTURA ESBELTA
}

Ing. Díaz, María Soledad, Ing. Schmidt, Erardo (*)

\section{RESUMEN}

El presente trabajo, se llevó a cabo en las instalaciones de la empresa TECNO TRANS, PyME ubicada en la ciudad de Oberá, Misiones, Argentina; que se dedica al mantenimiento y la reparación de todo tipo de transformadores monofásicos, trifásicos rurales y de distribución, así como el mantenimiento in situ de subestaciones transformadoras.

En este contexto, el objetivo principal del mismo se enfocó en la implementación de técnicas y herramientas propias de $\mathrm{Ma}$ nufactura Esbelta o Lean Manufacturing.

La Manufactura Esbelta busca implantar una filosofía de Mejora Continua que le permita a las empresas reducir sus costos, mejorar los procesos y eliminar los desperdicios para aumentar la satisfacción de los clientes y mantener el margen de utilidad. Consiste en varias herramientas que ayudan a eliminar todas las operaciones que no le agregan valor al producto, servicio y/o procesos, incrementando el valor de cada actividad y eliminando lo que no se requiere, con el fin de reducir desperdicios y mejorar las operaciones.

La incorporación de las diferentes técnicas en la empresa TECNO TRANS se desarrolló por etapas y una vez concluida la implementación de cada herramienta, se procedió a evaluar los resultados obtenidos.

El proceso de incorporación comenzó con el reconocimiento y documentación de la situación actual de la empresa: áreas de trabajo y procesos; se elaboró una propuesta de implementación de las técnicas de Manufactura Esbelta, estableciendo indicadores que permitan la evaluación de los resultados y la cuantificación de beneficios obtenidos por la empresa en su funcionamiento. La aplicación de algunas herramientas, permitió la obtención resultados satisfactorios y muy prometedores para la firma.

PALABRAS CLAVE: Manufactura Esbelta; PyME; Optimización de procesos; Mejora continua.

\section{LA EMPRESA}

TECNO TRANS, es una empresa unipersonal, que nace de una idea en el año 1990, cuando en la Provincia de Misiones no existía quien brinde el servicio de reparación y mantenimiento de transformadores monofásicos rurales y trifásicos rurales y de distribución, convirtiéndose en la principal fortaleza de la organización. Más de 20 años de experiencia avalan su trayectoria, convirtiéndola en la empresa referente en el rubro en la región. 
¿QUÉ ES LA MANUFACTURA ESBELTA O LEAN MANUFACTURING?

Es una filosofía de Mejora Continua, constituida por una serie de herramientas, las cuales permiten alcanzar los siguientes resultados: Incrementar el valor de cada actividad, Eliminar operaciones que no agregan valor al producto y/o servicio, Mantener el margen de utilidad de las organizaciones que la emplean, Aumentar la satisfacción del cliente, Eliminar desperdicios (Transporte, demoras, Inventarios, mala calidad, sobreproducción, etc.), Mejorar los procesos y Disminuir los costos.

\section{HERRAMIENTAS DE LA MANU- FACTURA ESBELTA}

Existen varias, como ser: Justo a Tiempo (JIT), Sistema KANBAN, 5"S", Mantenimiento Productivo Total (TPM), Productividad Efectiva de Equipos (PTEE), Verificación de Procesos (JIDOKA), Dispositivos para prevenir errores (POKA YOKE), Indicador Visual (ANDON), Cambio rápido de modelo (SMED), $\mathrm{Me}^{-}$ jora Continua (KAIZEN).

El presente trabajo se centró en la implementación de la herramienta $5^{\prime} \mathrm{S}$, este concepto se refiere a la creación y mantenimiento de áreas de trabajo más limpias, más organizadas y más seguras, es decir, se trata de imprimirle mayor "calidad de vida" al trabajo. Las 5'S son: Clasificar, organizar o arreglar apropiadamente (Seiri), Ordenar (Seiton), Limpieza (Seiso), standarizar (Seiketsu) y Disciplina (Shitsuke).

Algunos de los beneficios que genera la estrategias de las 5'S son: Mayores niveles de seguridad que redundan en una mayor motivación de los empleados, Mayor calidad, Tiempos de respuesta más cortos, $\mathrm{Au}-$ menta la vida útil de los equipos, Genera cultura organizacional y Reducción en las pérdidas y mermas por producciones con defectos.

\section{METODOLOGÍA Y PROCESO DE IMPLEMENTACIÓN}

Se elaboró un Plan de Trabajo, el cual se empleó como instrumento de planificación y sistematización de la información de modo de tener una visión global del trabajo a realizar, como ser objetivos, metas, actividades, responsables y tiempos, cuyo objetivo principal general se enfocó en la implementación de técnicas de Manufactura Esbelta; la cual se desarrolló por etapas:

ETAPA 1: Diagnóstico de la situación actual de la empresa (Procesos y actividades).

ETAPA 2: Capacitación del personal a través de charlas acerca de la técnica y la herramienta a implementar (entrega de material didáctico de soporte).

ETAPA 3: Implementación por fases de la herramienta " $5 \mathrm{~S}$ ", tendiente a mejorar los procesos, el manejo de los recursos y la participación del personal: Encuesta personal y anónima, para evaluar el conocimiento adquirido acerca del tema a implementar, Implementación de casa una de las 5 "S" por sector, efectuando un seguimiento continuo, Elaboración de Planillas para la recolección de información, para las distintas actividades y sectores dentro del proceso productivo, Creación de una base de datos digital para la carga y manejo de información para los sectores productivos y administrativos, tendiente a estandarizar el flujo de información, Desarrollo e implementación de una tarjeta de control (Kanban de producción), Registro fotográfico antes y después de la implementación de la herramienta 5"S". 
ETAPA 4: Evaluación de los resultados obtenidos: Los resultados se evaluaron a través de Indicadores de Gestión (Tabla 1) y por comparación de los registros fotográficos (Ilustración 1).
Cambio cultural en la forma de pensar y actuar por parte de todos los integrantes de la empresa.

\begin{tabular}{|ccccc|}
\hline \multicolumn{5}{c|}{ INDICADORES DE GESTIÓN } \\
\hline Nombre & Procedimiento de cálculo & $\begin{array}{c}\text { Uni } \\
\text { dad } \\
\text { de toma de } \\
\text { datos }\end{array}$ & $\begin{array}{c}\text { Frecuencia } \\
\text { de análisis }\end{array}$ \\
\hline $\begin{array}{c}\text { Eficiencla de } \\
\text { cada etapa }\end{array}$ & $\frac{\text { Tiempo empleado por cada etapa }}{\text { Tiempo estimado par a cada etapa }} \times 100$ & $\%$ & Diaria & Mensual \\
\hline $\begin{array}{c}\text { Eficiencia en el } \\
\text { armado }\end{array}$ & $\frac{N^{\circ} \text { de Transf.armados por semana }}{N^{\circ} \text { de Transf.planificados por semana }} \times 100$ & $\%$ & Semanal & Mensual \\
\hline $\begin{array}{c}\text { Eficacia en la } \\
\text { entrega }\end{array}$ & $\frac{\text { Pedidos realizados por los clientes }}{\text { Transformadores entregados }} \times 100$ & $\%$ & Semanal & Mensual \\
\hline $\begin{array}{c}\text { Tiempo de } \\
\text { armado de un } \\
\text { transformador }\end{array}$ & $\frac{\text { Tiempo empleado en el armado }}{\text { Tiempo estimado de armado }} \times 100$ & $\%$ & Diaria & Mensual \\
\hline
\end{tabular}

Tabla 1. Indicadores de Gestión. Fuente: TECNO TRANS, 2014.

\section{CONCLUSIONES}

Con la implementación parcial de la herramienta " $5 \mathrm{~S}$ " se pudieron obtener los siguientes resultados en el corto plazo: Eliminación de desperdicios, Incremento de la seguridad e higiene en los distintos centros de trabajo, Mayor espacio útil en planta y oficina, Reducción del tiempo de acceso al material y las herramientas de trabajo, Se mejoró la accesibilidad a la Información (Trabajos realizados, ventas, clientes y proveedores), Se mejoró el control visual de stocks de repuesto y elementos de producción y fundamentalmente se logró un
Se logró superar la resistencia al cambio por parte del personal, lo cual mejoró el tiempo de ejecución de cada una de las etapas.

A pesar de esto se han logrado resultados muy alentadores en el corto plazo en todas las áreas de la empresa, augurando un futuro prometedor para la firma, la que actualmente está encaminada a profundizar los cambios derivados de la implementación de otras técnicas conducentes a lograr la Manufactura Esbelta. 

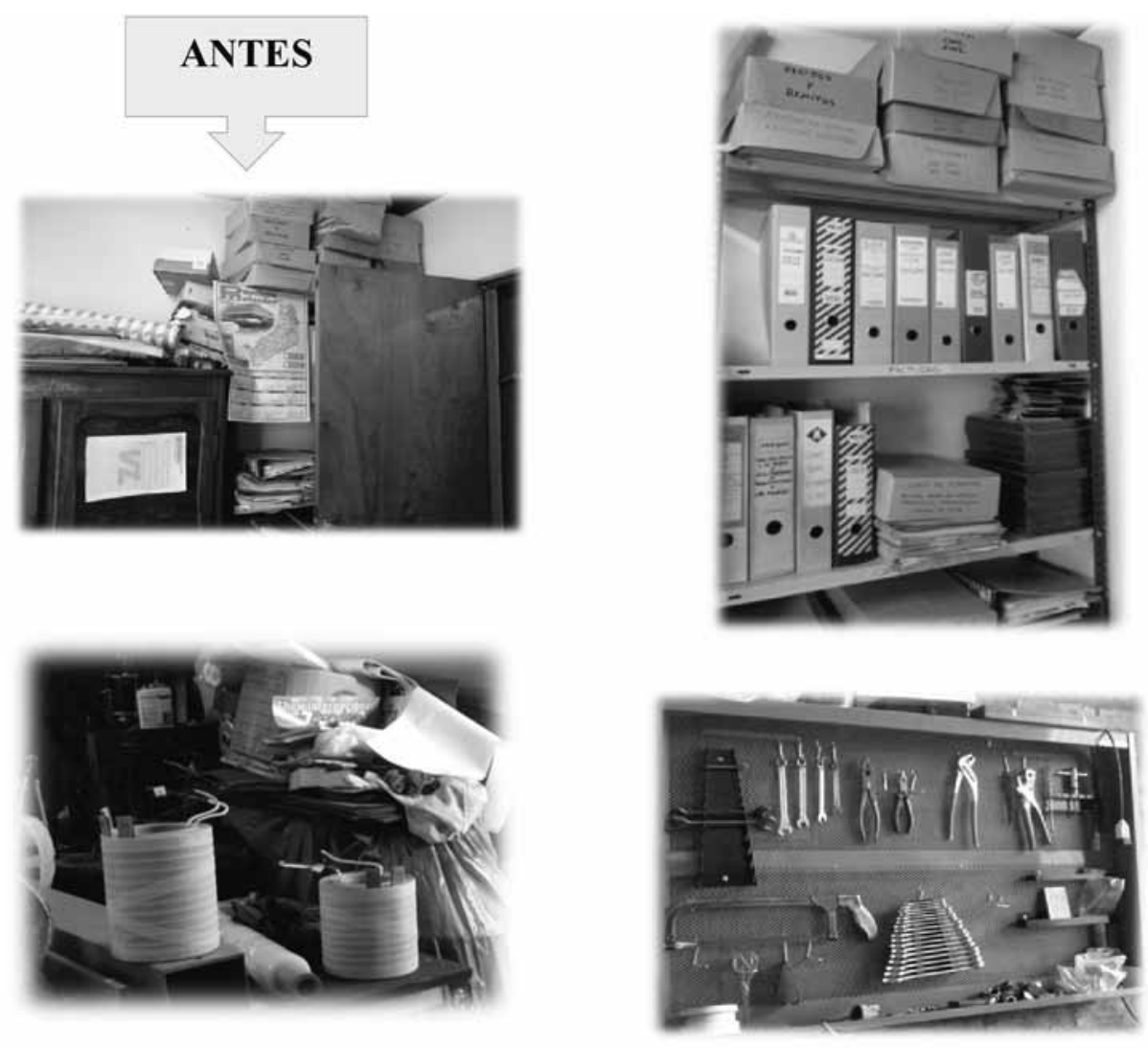

\section{DESPUÉS}

Ilustración 1. Antes y después de la implementación de la herramienta “5 S". Fuente: TECNO TRANS, 2014. 